\title{
The state of social science research on COVID-19
}

\author{
Yan-Li Liu ${ }^{1}$ (D) Wen-Juan Yuan ${ }^{1} \cdot$ Shao-Hong Zhu ${ }^{1}$
}

Received: 14 February 2021 / Accepted: 3 November 2021 / Published online: 21 November 2021

(c) Akadémiai Kiadó, Budapest, Hungary 2021

\begin{abstract}
Research on COVID-19 has proliferated rapidly since the outbreak of the pandemic at the end of 2019. Many articles have aimed to provide insight into this fast-growing theme. The social sciences have also put effort into research on problems related to COVID-19, with numerous documents having been published. Some studies have evaluated the growth of scientific literature on COVID-19 based on scientometric analysis, but most of these analyses focused on medical research while ignoring social science research on COVID-19. This is the first scientometric study of the performance of social science research on COVID-19. It provides insight into the landscape, the research fields, and international collaboration in this domain. Data obtained from SSCI on the Web of Science platform was analyzed using VOSviewer. The overall performance of the documents was described, and then keyword co-occurrence and co-authorship networks were visualized. The six main research fields with highly active topics were confirmed by analysis and visualization. Mental health and psychology were clearly shown to be the focus of most social science research related to COVID-19. The USA made the most contributions, with the most extensive collaborations globally, with Harvard University as the leading institution. Collaborations throughout the world were strongly related to geographical location. Considering the social impact of the COVID-19 pandemic, this scientometric study is significant for identifying the growth of literature in the social sciences and can help researchers within this field gain quantitative insights into the development of research on COVID-19. The results are useful for finding potential collaborators and for identifying the frontier and gaps in social science research on COVID-19 to shape future studies.
\end{abstract}

Keywords COVID-19 $\cdot$ Social science $\cdot$ VOSviewer $\cdot$ Scientometric $\cdot$ Visualization

Yan-Li Liu

liuyanli@hmc.edu.cn

1 Library of Hangzhou Medical College, 8 Yikang Street, Lin'an District, Hangzhou 311399,

People's Republic of China 


\section{Introduction}

\section{Background}

The COVID-19 pandemic has been spreading throughout the world for almost two years. There is no doubt that all of society is heavily affected by this outbreak, including the domains of public health, education, economics, and industry. Meanwhile, most people in the world are suffering as well. Numerous studies from different perspectives have been made since the beginning of the epidemic, and there are many studies in the social sciences as well as in the field of medicine.

The number of publications on COVID-19 is growing rapidly every day as the pandemic continues, so it is necessary to help researchers assess the research trends and topics on this issue. Scholars have already tried to do so. Valuable research patterns from publications and developments related to the coronavirus and COVID-19 were identified based on scientometric indicators (Sahoo \& Pandey, 2020). The most productive countries and the international scientific collaboration on coronavirus, as well as the proportion and typology of open accessibility to the relevant publications, have also been identified (Belli et al., 2020). The current hotspots and future directions for COVID-19 research-including clinical features studies, pathological findings and therapeutic design, care facilities preparation and infection control, and maternal, perinatal, and neonatal outcomes-have been tracked by bibliometric analysis (Zyoud \& Al-Jabi, 2020). Based on a bibliometric analysis using VOSviewer for publications on COVID-19, disease treatment, spike proteins, and vaccines were found to be future hotspots (Yu et al., 2020). These studies have paved the way for a better understanding of the progress in research and developments in combatting COVID19 , especially in the field of medicine.

However, most of these studies have focused on the medical aspects while the social science research on COVID-19 was largely ignored. A scientometric analysis of the literature related to COVID-19 in the domain of the social sciences is still lacking. This is thus the first scientometric study of COVID-19 related to social science research performance, and it provides insight into the landscape, research fields, and international collaborations.

\section{Objective}

To find out what topics are considered in the social science fields, we aimed to investigate the state of social science research on COVID-19 by scientometric analysis, including scientific collaborations and impact, current research fields, and hot topics. The study of the state of social science research on antimicrobial resistance (AMR) published in Social Science \& Medicine provided a precedent; it found that social science research constituted a negligible share of total academic contributions, and the impact of social science research on the scientific discourse on AMR was both peripheral and thinly spread (Frid-Nielsen, Rubin, \& Baekkeskov, 2019). The aim of this study was to focus on the landscape of research on COVID-19 in the domain of the social sciences.

By employing scientometric mapping, we provide a broad view of divisions in the social science research on COVID-19. Six research fields were extracted, among which mental health and psychology had attracted the most attention throughout the world. The hotspots fell mostly within this theme too. As a nexus for the COVID-19 pandemic, the USA has made the most contributions in the social sciences on this topic, with the most 
extensive collaborations globally. Harvard University was the most productive source of social science research as an institution. Collaborations throughout the world were strongly related to geographical location. These findings can help researchers within the social sciences gain quantitative insights into the development of research related to COVID-19. The results are useful for finding potential collaborators and for identifying research frontiers and gaps to shape directions for future inquiry.

\section{Methods}

This study mainly followed the generic framework for scientometric mapping research ( $\mathrm{Li}$, Goerlandt, \& Reniers, 2021). Based on the research purpose, the data set was collected and analyzed as follows.

\section{Data source and retrieval strategies}

The objective of this study was focused on the social science field, and we conducted a search in the Social Science Citation Index (SSCI) available at the Web of Science (WoS) platform. The search terms included "COVID-19" OR "*nCoV" OR "Sars-CoV-2" OR "new coronavirus" OR "coronavirus disease 2019" OR "severe acute respiratory syndrome coronavirus-2" OR "novel coronavirus" OR "coronavirus 19," which were restricted to the title, abstract, or keywords. The search results were then refined by document type, and only articles were selected. Since the epidemic began at the end of 2019, documents published before 2019 were excluded.

\section{Data cleaning}

The data downloaded from WoS are not always "clean." There may be some duplicate records or key information missing. The records retrieved according to the criteria above were input into HistCite (Garfield et al., 2006) for cleaning. The cleaned data were then exported as a CSV file for the final analysis and visualization.

\section{Data analysis and visualization}

VOSviewer (van Eck \& Waltman, 2010, 2017) was used to construct and visualize the scientometric networks. The CSV file was directly input into this software. Most scientometric methods are relation-based analyses, which can be divided into three categories: citation relations, word co-occurrence, and co-authorship relations (Li et al., 2021). This study was performed based on all three types of relationships. The citation relation methods can provide insight into clusters of connected ideas and can be further divided into direct citation, bibliographic coupling, and co-citation relations (Klavans \& Boyack, 2017). Word co-occurrence analysis can be used to reveal research topics and trends and is based on the number of co-occurrences of two words appearing in the same publication, abstracts, or keywords. Co-authorship analysis, also known as author collaboration, can reflect the social structure and social relationships of the scientific domain (Li et al., 2021). There are three levels of co-authorship: authors analysis, institute or city analysis, and country/region analysis. 
Table 1 Top 10 most productive countries for COVID-19 SSCI articles

\begin{tabular}{llcc}
\hline Ranking & Country & Records & Citations \\
\hline 1 & USA & 3217 & 10,657 \\
2 & China & 1229 & 10,074 \\
3 & England & 1202 & 5990 \\
4 & Italy & 726 & 2867 \\
5 & Australia & 676 & 2993 \\
6 & Canada & 630 & 3289 \\
7 & Spain & 550 & 1544 \\
8 & Germany & 436 & 1177 \\
9 & Brazil & 320 & 1126 \\
10 & France & 236 & 1040 \\
\hline
\end{tabular}

Table 2 Top 10 most productive institutions for COVID-19 SSCI articles

\begin{tabular}{llcc}
\hline Ranking & Institution & Records & Citations \\
\hline 1 & Harvard Univ & 182 & 624 \\
2 & Univ Oxford & 123 & 969 \\
3 & Univ Toronto & 120 & 1039 \\
4 & UCL & 101 & 973 \\
5 & Johns Hopkins Univ & 90 & 940 \\
6 & Univ Melbourne & 90 & 328 \\
7 & Kings Coll London & 87 & 977 \\
8 & Huazhong Univ Sci \& Technol & 86 & 1288 \\
9 & Yale Univ & 86 & 414 \\
10 & Wuhan Univ & 81 & 1900 \\
\hline
\end{tabular}

The analysis proceeded as follows. First, the global distribution of the SSCI articles on COVID-19 was described. Then, keyword co-occurrence analysis and visualization were conducted to demonstrate the research fields and hot topics. Finally, co-authorship analysis at the country/region and institutional levels was carried out.

\section{Results}

\section{Global distribution of SSCl articles on COVID-19}

In total, 10,033 articles related to COVID-19 published from 2019 were identified in SSCI on January 28, 2021. There was only one article found for 2019, which was published before the outbreak of COVID-19; it was therefore excluded from our study. The final data after cleaning contained 9,954 documents. The overall distribution characteristics are presented below.

The top 10 most productive countries and institutions for COVID-19 SSCI articles are listed in Tables 1 and 2, respectively. A total of 3217 articles from the USA have been cited 10,657 times throughout the world, followed by China with 1229 articles and 10,074 citations and England with 1202 articles and 5990 citations (Table 1). A total of 182 articles from Harvard University have been cited 624 times, followed by the University of Oxford, 
with 123 articles and 969 citations, and the University of Toronto with 120 articles and 1039 citations.

The top 10 most-cited journals and articles contributing to COVID-19 research are listed in Tables 3 and 4, respectively. A total of 1606 journals were found to publish articles on COVID-19 in the SSCI, and 1115 articles were published in the top 10 cited journals, with 11,403 citations. The International Journal of Environmental Research and Public Health has published 679 articles, with 3531 citations (Table 3). As we can see in Table 3, most of the 10 journals are related to public health, mental health, or psychology. As shown in Table 4, the most-cited article was written by CY Wang, published in the International Journal of Environmental Research and Public Health, which is also the most-cited journal. The sources for the other nine articles were also among the top 10 cited journals (Tables 3, 4).

\section{Keyword co-occurrence}

We conducted keyword co-occurrence analysis to reveal the research fields and hot topics related to COVID-19. The minimum number of occurrences of a keyword provided by the authors was set to 15. For all of the articles around the theme of COVID-19, terms used in the retrieval were excluded from the analysis, so the relationships among the remaining keywords could be demonstrated more clearly. Variants of the different keywords were merged. Ultimately, 291 keywords were enrolled in the analysis. The keyword co-occurrence network is shown in Fig. 1, and the top 10 keywords are displayed in Table 5. More details about the clusters can be found in Table 6 .

As shown in Fig. 1, seven keyword clusters were obtained by co-occurrence analysis. The size of the nodes represents the occurrence counts, and the links between two nodes represent their co-occurrence in the same document. The closer two nodes are to each other, the larger the number of co-occurrences for these two keywords.

Cluster 1: Public Health.

The biggest cluster of keywords, shown in red, includes 110 items, which represent the research field of public health, concerning the epidemiology of COVID-19, social distancing, health policy, ethics, gender, and so on (Table 6). This research field reveals most of the social problems related to COVID-19.

Table 3 Top 10 most-cited journals contributing to COVID-19 research

\begin{tabular}{llcc}
\hline Ranking & Journal & Records & Citations \\
\hline 1 & International Journal of Environmental Research and Public & 679 & 3531 \\
& Health & & 1705 \\
2 & Psychiatry Research & 65 & 1169 \\
3 & Brain Behavior and Immunity & 14 & 932 \\
4 & JAMA Network Open & 22 & 828 \\
5 & Lancet Global Health & 14 & 752 \\
6 & Lancet Public Health & 13 & 695 \\
7 & International Journal of Mental Health and Addiction & 43 & 652 \\
8 & Lancet Psychiatry & 8 & 597 \\
9 & Frontiers in Public Health & 238 & 542 \\
10 & Nature Human Behaviour & 19 & \\
\hline
\end{tabular}




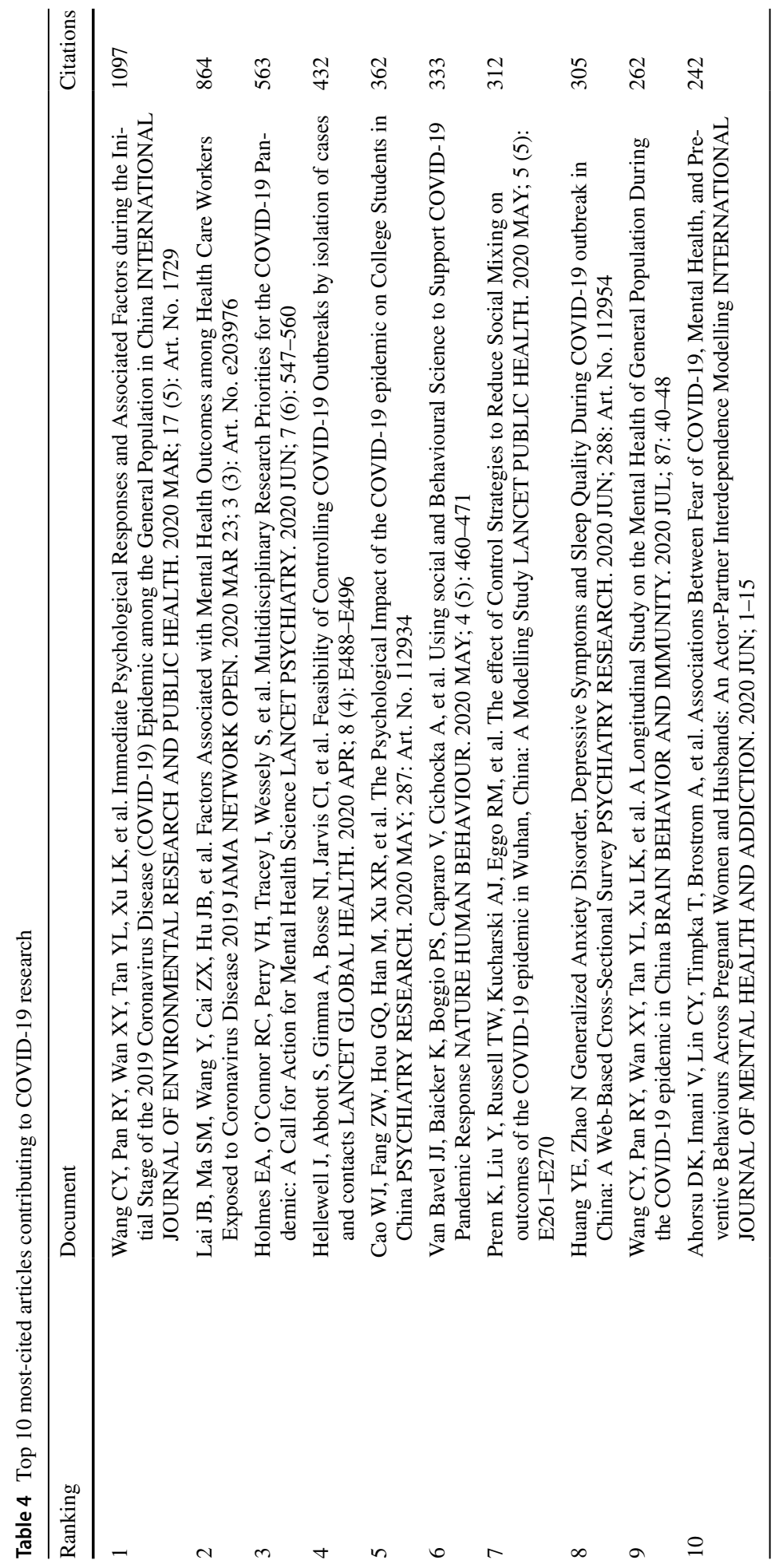




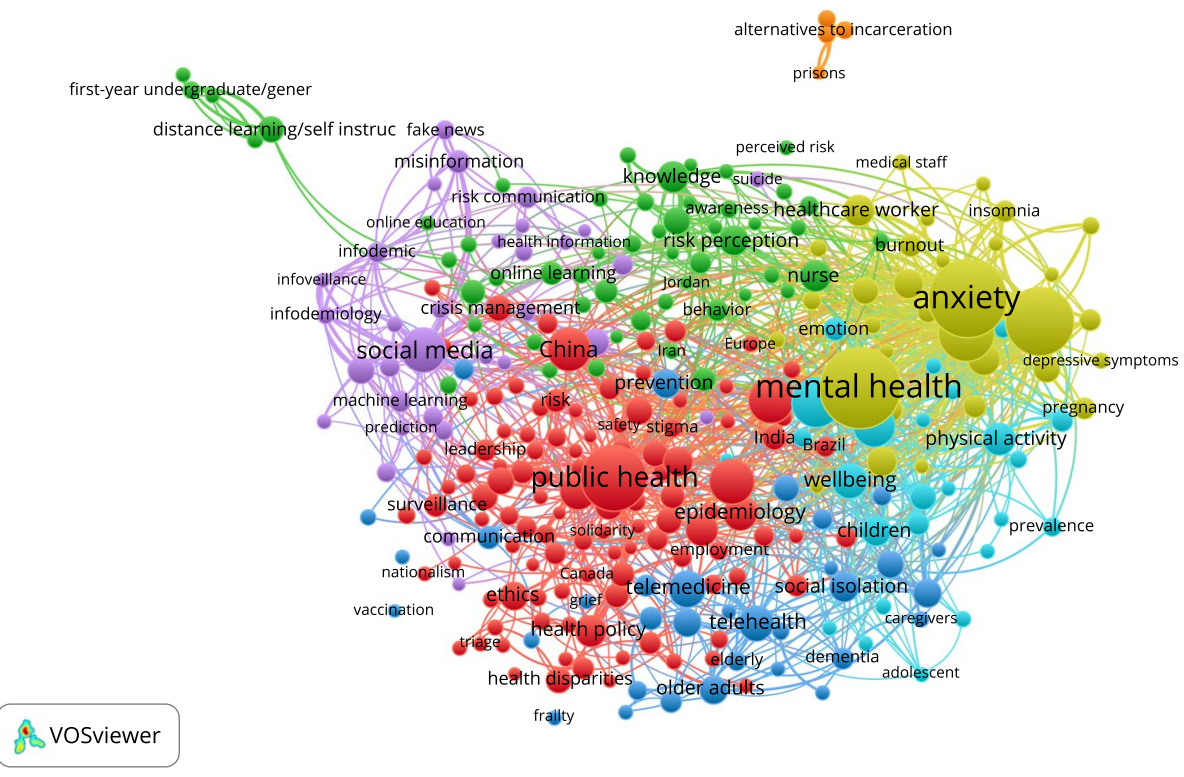

Fig. 1 Keyword co-occurrence network. The size of the nodes represents the occurrence counts. The links between two nodes represent their co-occurrence in the same document. The closer two nodes are to each other, the larger the number of co-occurrences for the two keywords

Table 5 Top 10 keywords

\begin{tabular}{llll}
\hline Ranking & Keyword & Cluster & Occurrence \\
\hline 1 & Mental health & 4 & 550 \\
2 & Anxiety & 4 & 503 \\
3 & Depression & 4 & 397 \\
4 & Public health & 1 & 355 \\
5 & Stress & 4 & 264 \\
6 & Lockdown & 6 & 202 \\
7 & Resilience & 1 & 174 \\
8 & Social media & 5 & 174 \\
9 & Social distancing & 1 & 169 \\
10 & China & 1 & 157 \\
\hline
\end{tabular}

Cluster 2: Health Literacy \& Education.

The green cluster represents the topic of health literacy in coping with this pandemic and concerns citizen's knowledge about the disease, risk perception, attitude, behavior, and so on.

Cluster 3: Telemedicine.

The blue cluster represents the research field of telemedicine, especially as delivered to special populations such as older adults and people with dementia, who are facing great problems in terms of social isolation and loneliness.

Cluster 4: Mental Health \& Psychology. 


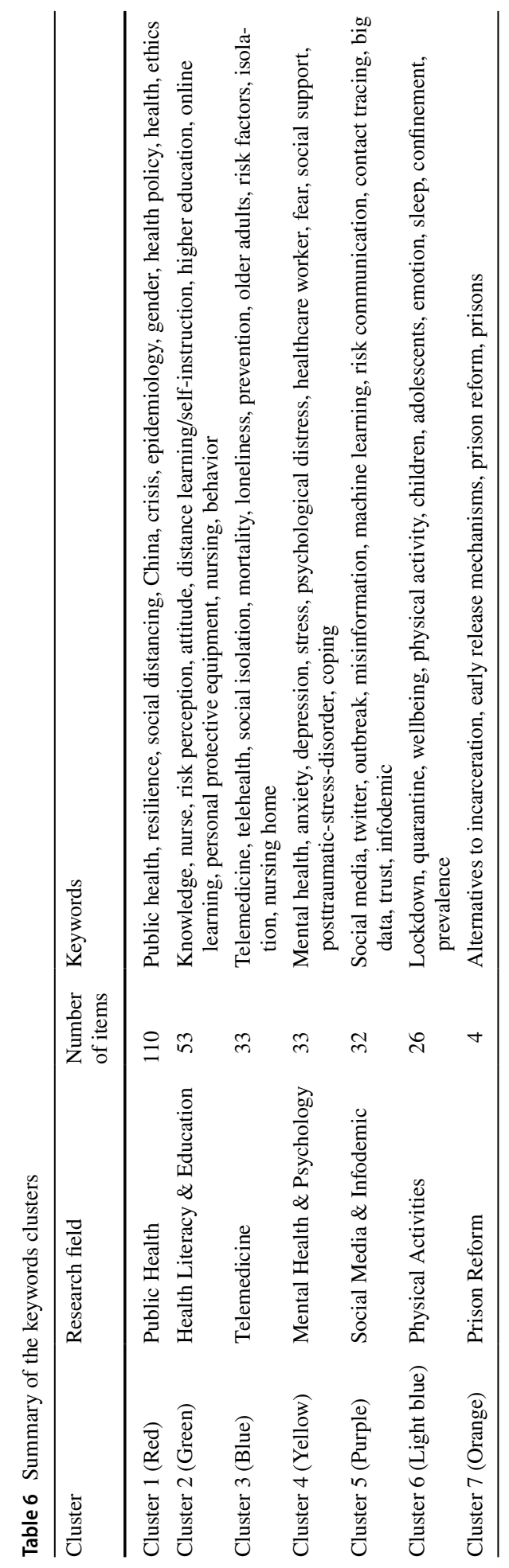


The yellow cluster represents the research field of mental health and psychology, which is the most active in the social sciences, concerning the mental and psychological states of healthcare workers. As indicated in Table 5, mental health, anxiety, and depression are the top three keywords found. Another keyword, stress, is the fifth, following public health.

Cluster 5: Social Media \& Infodemics.

The purple cluster represents the research field of social media and infodemics, which analyzes health information related to COVID-19 on social media using methods like content analysis, network analysis, and machine learning. This cluster is a relatively new subject, which can be called infodemiology, and is based on the concern raised by the spread of disinformation and misinformation on health since the outbreak of COVID-19.

Cluster 6: Physical Activities.

The light blue cluster represents the research field of physical activities during the epidemic, concerning the special period of quarantine or lockdown.

Cluster 7: Prison Reform.

The orange cluster has only four items, representing a small research field that is relatively independent. This cluster illustrates the study on prison reform in the COVID-19 environment.

\section{Co-authorship of countries/regions and institutions}

To investigate the social relations across countries/regions and institutions throughout the world, we conducted co-authorship analysis. The network visualizations for country/region co-authorship and institutional co-authorship are shown in Figs. 2 and 3, respectively.

We set the minimum number of documents for a country/region to five and the minimum number of citations for a country/region as zero. Of the 167 countries/regions, 109 met these thresholds. Six country/regione clusters were obtained, and these are shown in Fig. 2. The size of the nodes represents the total link strength of the country/region. The more links the node has, the larger the size of the node. The stronger the link between two nodes is, the thicker the line between them. There are 2,228 links, and the total link strength is 10,934 .

The cluster shown in red has the most items and contains 27 countries/regions, including Italy, Germany, the Netherlands, and Sweden. Most of these countries/regions are in Europe. The green cluster has 26 items, including France, India, Iran, Egypt, Japan, and Saudi Arabia. The blue cluster has 22 items, and most of the countries are in Africa including South Africa, Nigeria, and Kenya. The yellow cluster contains countries in the Americas, including the USA, Brazil, Mexico, and Peru, as well as Spain in Europe. The purple cluster contains 10 items, including China, Australia, Canada, Singapore, and Pakistan. The light blue cluster is the smallest and contains nine items, with England, Switzerland, New Zealand, Scotland, and Ireland as the main countries.

As indicated in Fig. 2, the USA has the most extensive collaborations with other countries/regions worldwide. It has 100 links, with a total link strength of 2231. It is followed by England with 98 links, and Canada and Germany with 81 links each. The main partners of the USA are England, China, and Canada. Geographic position appears to be an important factor influencing the collaborations among countries/regions. The more productive a country/region is, the wider its network of collaborations.

We set the minimum number of documents for an institution to 20 and the minimum number of citations an institution to 40 . Of the 9484 institutions, 184 met these thresholds. Six clusters of institutions were obtained, as shown in Fig. 3. The size of the nodes 


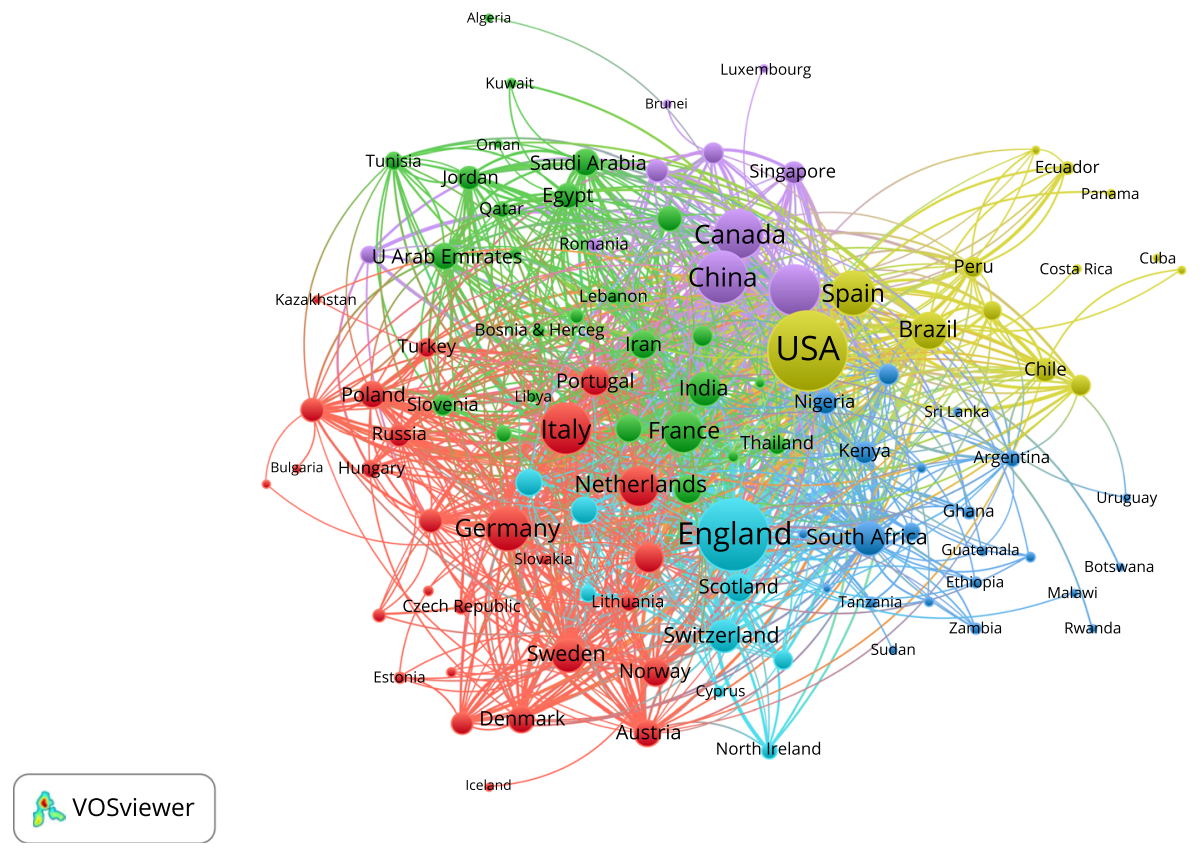

Fig. 2 Country/region co-authorship network. The size of the nodes represents the total link strength of the country/region. The more links the node has, the larger the size of the node. The stronger the link between two nodes is, the thicker the line between them

represents the total link strength of the institution. The more links the node has, the larger the size of the node. The stronger the link between two nodes is, the thicker the line between them. There are 2609 links, and the total link strength is 4224 .

The largest cluster, shown in red, has 56 items, mainly containing universities in the USA, including Harvard University, the University of Michigan, Yale University, and Johns Hopkins University.

The second cluster, in green, has 37 items, mainly containing institutions in England, including University College London, University of Oxford, King's College London, and London School of Hygiene \& Tropical Medicine.

The blue cluster contains 29 items, mainly including institutions in Australia such as the University of Melbourne, University of Sydney, Monash University, and University of Queensland.

The yellow cluster contains 26 items. Most of these institutions are in Italy, and the others are scattered throughout Europe including in England, France, Sweden, and Spain. The institutions involved include the University of Milan, University of Padua, Sapienza University of Rome, University of Valencia, University of Paris, and the University of Cambridge.

The purple cluster contains 24 items, which are mainly institutions in China such as Wuhan University, Huazhong University of Science and Technology, Zhejiang University, and Chinese University of Hong Kong. 


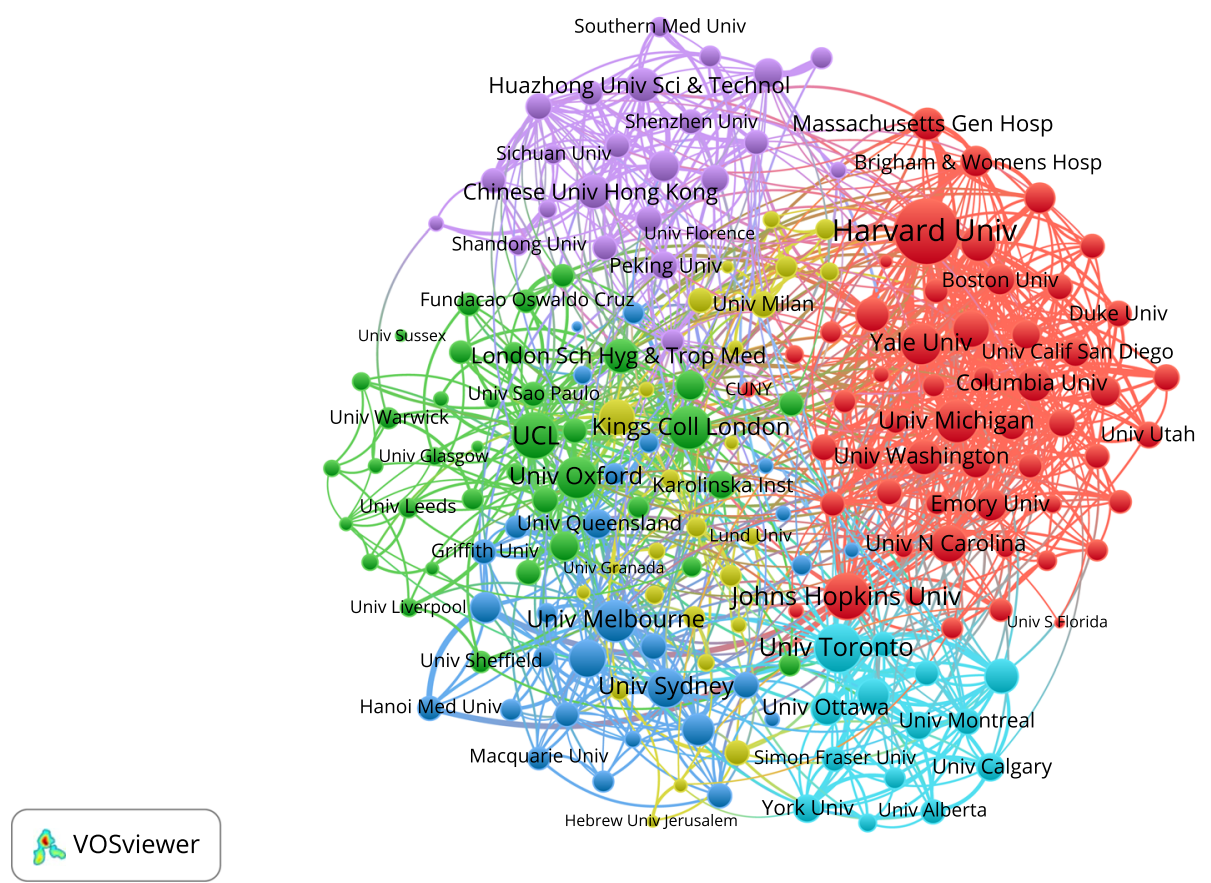

Fig. 3 Institution co-authorship network. The size of the nodes represents the total link strength of the institution. The more links the node has, the larger the size of the node. The stronger the link between two nodes is, the thicker the line between them

The smallest cluster is light blue with 14 items, mainly containing institutions in Canada such as the University of Toronto, University of British Columbia, University of Ottawa, and McGill University.

As suggested by Fig. 3, Harvard University appears to have the most extensive collaboration network with other institutions all over the world. It has 105 links, with a total link strength of 305. It is followed by University College London with 75 links and University of Oxford with 81 links. The main partners of Harvard University are Massachusetts General Hospital and Brigham and Women Hospital, both of which are affiliated teaching hospitals of Harvard University. Collaborations among institutions are more likely to happen within the same country/region. Transnational collaborations are more common in Western Europe.

\section{Discussion}

\section{Social science research fields and hot topics related to COVID-19}

We found six research fields from the analysis of keyword co-occurrence: public health, health literacy \& education, telemedicine, mental health \& psychology, social media \& infodemics, physical activities, and prison reform. This suggests that people's emotional 
experiences, as well as physical activities, during the COVID-19 pandemic are considered within the realm of social science research.

The most frequent keyword related to COVID-19 is mental health. Anxiety, depression, and stress are also among the top five keywords. All of the above keywords belong to the field of mental health \& psychology. This field appears to be dominant in the social science research related to COVID-19. The most-cited article was written by Wang et al. (2020) and was also about psychology in the pandemic (Table 4). In the fight against COVID-19, unprecedented pressure has been placed on healthcare workers around the world. Many people are also suffering under great psychological burdens and experiencing anxiety as a result of the pandemic. The psychological wellbeing of these people has therefore attracted a lot of attention in the field of social science.

The topics in public health range widely and include most of the social problems related to health such as ethics (Martinez-Martin et al., 2020), racism (Cheah et al., 2020; Wilby et al., 2021), and health equity (Azria et al., 2020). As an important measure for protecting public health during COVID-19, social distancing is frequently discussed. China, as the first country to report COVID-19, ranks tenth in the keywords (Cheah et al., 2020). Sharing the experience of China with the pandemic appears to be considered valuable.

The COVID-19 crisis has had profound effects on education. Many students have not been able to continue regular schooling and have had to rely on remote learning because of COVID-19 quarantines. Knowledge about health, especially risk perception, has been proposed for delivery to the public. Health literacy \& education is thus one of the key themes related to COVID-19 (Abdel-Latif, 2020; Bellini et al., 2020).

It should be noted that everyday life has been affected by social media for years. During COVID-19, social media has had both positive and negative effects on the public (Mano, 2020). Along with useful information and social support, social media has brought the public vast quantities of fake news, disinformation, or misinformation. This issue gives meaning to the word "infodemic," which was coined by Eysenbach (2002), and is closely related to infodemiology and infoveillance (Eysenbach, 2020).

As a result of quarantines during the COVID-19 outbreak, people's physical activities have also been deeply affected. The results of survey by Lesser and Nienhuis (2020) indicated that physical activities had a different impact on active and inactive individuals. The well-being outcomes in inactive individuals were strongly associated with physical activity.

It is also noteworthy that technologies such as telemedicine (Colbert et al., 2020), telehealth (Mehta et al., 2021), and telepsychiatry (O'Brien \& McNicholas, 2020) have been adopted to solve the difficulties of special populations, especially those in social isolation.

It should not be ignored that COVID-19 is especially awful in prisons due to the crowded conditions and lack of availability of clean water, soap, and ventilation; access to healthcare is also inadequate (Quinn, 2020). Some researchers have aimed to promote prison reform to curb the transmission of COVID-19 in the prison population (Reinhart \& Chen, 2020).

\section{Scientific contributions and social relations of countries/regions and institutions}

The contribution of a country/region is somewhat related to the state of the COVID-19 crisis in that country/region. Collaborations among countries/regions or institutions are strongly linked with their geographic position, considering that social problems often have regional characteristics. People from different parts of the world are in different situations and their concerns vary accordingly. However, another bibliometric analysis of research 
on traditional Chinese medicine for COVID-19 revealed a similar phenomenon-namely, that geographical distance was the main factor affecting cooperation in regional scientific research, and researchers were more inclined to seek cooperation within the same region (Yang et al., 2020).

It is remarkable that the USA has made the most contributions in social science research related to COVID-19. The USA produces the most social science research in the world (ILOVEPHD, 2021), so it's not unexpected that it would also contribute the most social science research related to COVID-19. The USA also has the most extensive collaborations throughout the world, and this is paralleled in the case of Harvard University at the institutional level. The USA is the most affected country in the world with the highest number of COVID-19 cases and deaths. Almost every part of daily life for people in this country has been disrupted by the novel coronavirus, and the flaws in various American systems have been made apparent (Melendez, 2021).

Although this study was based on social science research, we found that Harvard University, as the most productive institution, also collaborated quite closely with hospitals. We suppose that the social research related to COVID-19 would, to a great extent, be about the health of the population. Because large-scale behavioral change is required in this crisis, and significant psychological burdens are placed on individuals, with the recommendations of epidemiologists and public health experts, insights from social and behavioral sciences can be useful for helping align human behavior with situational requirements (Bavel et al., 2020).

\section{Implications}

The results of this study will be useful for social science researchers to find potential collaborators and to identify research frontiers and gaps in research on COVID-19 to shape future directions for inquiry. It is revealed in this study that collaborations among countries/regions or institutions are strongly linked with their geographic position. Maybe social science researchers can search for more potential collaborators throughout the world thanks to the results of this study. The COVID-19 pandemic is much more than a health crisis. It is a human, economic, and social crisis. Beyond the research fields and hot topics found in this study, there remains much more for social science researchers to study further, such as the impact of COVID-19 on finance, economics, social policy, public events, employment, tourism, librarianship, and so on. A number of critical social and economic issues, as well as the corresponding solutions, urgently need to be solved. Improvements to public health systems and strategies for confronting future pandemics are also in great need of study.

\section{Limitations}

Several limitations of this study should be resolved in future scientometric research. First, only the Web of Science database was used to search for documents; other data sources such as Scopus, Google Scholar, Index Medicus, or Microsoft Academic Search were neglected. Data searched from these sources may be different in terms of document or citation counts, thus resulting in different conclusions. Second, only one scientometric tool was used for analysis in this study. There are more than 25 scientometric tools available (Li, 2017), but none are perfect. Analysis performed by different tools may yield different 
results, and a wider range of tools could be applied to cover the deficiencies of this study. Nonetheless, this study elaborates on the scientometric analysis of the theme, and we believe that it provides a sound overview of the landscape of international social science research on COVID-19.

Acknowledgements We thank LetPub (www.letpub.com) for its linguistic assistance during the preparation of this manuscript.

Author contributions Yan-Li Liu: Conceptualization, Formal analysis, Writing-Original Draft. Wen-Juan Yuan: Methodology, Investigation, Visualization. Shao-Hong Zhu: Data curation, Writing-Review \& Editing.

Funding No funding was received for conducting this study.

Data Availability All data are available on request.

\section{Declarations}

Conflict of interest The authors declare that there is no conflict of interest.

Consent for publication All authors have approved the version to be published.

\section{References}

Abdel-Latif, M. M. M. (2020). The enigma of health literacy and COVID-19 pandemic. Public Health, 185, 95-96.

Azria, E., Sauvegrain, P., Blanc, J., Crenn-Hebert, C., Fresson, J., Gelly, M., et al. (2020). Systemic racism and health inequalities, a sanitary emergency revealed by the COVID-19 pandemic. Gynecologie Obstetrique Fertilite \& Senologie, 48(12), 847-849.

Bavel, J., Baicker, K., Boggio, P. S., Capraro, V., Cichocka, A., Cikara, M., et al. (2020). Using social and behavioural science to support COVID-19 pandemic response. Nature Human Behaviour, 4(5), 460-471.

Belli, S., Mugnaini, R., Baltà, J., \& Abadal, E. (2020). Coronavirus mapping in scientific publications: When science advances rapidly and collectively, is access to this knowledge open to society? Scientometrics, 124(3), 2661-2685.

Bellini, M. I., Pengel, L., Potena, L., \& Segantini, L. COVID-19 and education: restructuring after the pandemic. TRANSPLANT INTERNATIONAL.

Cheah, C. S. L., Wang, C., Ren, H., Zong, X., Cho, H. S., \& Xue, X. (2020). COVID-19 racism and mental health in Chinese American families. Pediatrics, 146, e20200218165.

Colbert, G. B., Verner Venegas-Vera, A., \& Lerma, E. V. (2020). Utility of telemedicine in the COVID-19 era. Reviews in Cardiovascular Medicine, 21(4), 583-587.

Eysenbach, G. (2002). Infodemiology: The epidemiology of (mis)information. The American Journal of Medicine, 113(9), 763-765.

Eysenbach, G. (2020). How to fight an infodemic: The four pillars of infodemic management. Journal Of Medical Internet Research, 22(6), e21820.

Frid-Nielsen, S. S., Rubin, O., \& Baekkeskov, E. (2019). The state of social science research on antimicrobial resistance. Social Science \& Medicine, 242, 112596.

Garfield, E., Paris, S. W., \& Stock, W. G. (2006). HistCiteTM: A software tool for informetric analysis of citation linkage. Nfd Information Wissenschaft Und Praxis, 57(8), 391-400.

Klavans, R., \& Boyack, K. W. (2017). Which type of citation analysis generates the most accurate taxonomy of scientific and technical knowledge? Journal of the Association for Information Science and Technology, 68(4), 984-998.

Lesser, I. A., \& Nienhuis, C. P. (2020). The Impact of COVID-19 on Physical Activity Behavior and WellBeing of Canadians. International Journal of Environmental Research and Public Health, 17, 389911.

Li, J. (2017). Scientometrics and knowledge network analysis. Capital University of Economics and Business Press. 
Li, J., Goerlandt, F., \& Reniers, G. (2021). An overview of scientometric mapping for the safety science community: Methods, tools, and framework. Safety Science, 134, 105093.

Mano, R. (2020). Social media and resilience in the COVID-19 crisis. Advances in Applied Sociology, 10(11), 454-464.

Martinez-Martin, N., Dasgupta, I., Carter, A., Chandler, J. A., Kellmeyer, P., Kreitmair, K., et al. (2020). Ethics of digital mental health during COVID-19: Crisis and opportunities. JMIR Mental Health, 7, e2377612.

Mehta, P., Stahl, M. G., Shull, M., Liu, E., Germone, M. M., Nagle, S., et al. (2021). Telehealth and nutrition support during the COVID-19 pandemic. Journal of the Academy of Nutrition and Dietetics, 121(1), 1953-1957.

Melendez, E. (2021). The impact of COVID-19 on The United States of America. Transdisciplinary Journal of Engineering \& Science, 12, 13-15.

O'Brien, M., \& McNicholas, F. (2020). The use of telepsychiatry during COVID-19 and beyond. Irish Journal Of Psychological Medicine, 37, 250-255.

Online document ILOVEPHD. Top 50 Countries in Social Science Research 2020. Retrieved September 24, 2021, from https://www.ilovephd.com/top-50-countries-in-social-science-research-2020/.

Quinn, E. (2020). COVID-19, Race, and the Case for Canadian Prison Reform.

Reinhart, E., \& Chen, D. L. (2020). Incarceration and its disseminations: COVID-19 pandemic lessons from Chicago's cook County Jail. HEALTH AFFAIRS, 39(8), 1412-1418.

Sahoo, S., \& Pandey, S. (2020). Evaluating research performance of Coronavirus and Covid-19 pandemic using scientometric indicators. ONLINE INFORMATION REVIEW, 44(7), 1443-1461.

Spring, H. (2020). Health literacy and COVID-19. Health Information and Libraries Journal, 37(3), $171-172$.

van Eck, N. J., \& Waltman, L. (2010). Software survey: VOSviewer, a computer program for bibliometric mapping. Scientometrics, 84(2), 523-538.

van Eck, N. J., \& Waltman, L. (2017). Citation-based clustering of publications using CitNetExplorer and VOSviewer. Scientometrics, 111(2), 1053-1070.

Wang, C., Pan, R., Wan, X., Tan, Y., Xu, L., Ho, C. S., et al. (2020). Immediate psychological responses and associated factors during the initial stage of the 2019 Coronavirus Disease (COVID-19) epidemic among the general population in China. International Journal of Environmental Research and Public Health, 17, 17295.

Wilby, K. J., De Chun, L., Ye, R., \& Smith, A. J. (2021). Students' experiences with racism during the COVID-19 pandemic. Academic Medicine, 96(1), E4.

Yang, K., Jin, X., Gao, Y., Xie, J., Liu, M., Zhang, J., et al. (2020). Bibliometric analysis of researches on traditional Chinese medicine for coronavirus disease 2019 (COVID-19). Integrative Medicine Research, 9(3), 100490.

Yu, Y., Li, Y., Zhang, Z., Gu, Z., Zhong, H., Zha, Q., et al. (2020). A bibliometric analysis using VOSviewer of publications on COVID-19. Annals of Translational Medicine, 8(13), 816.

Zyoud, S. E. H., \& Al-Jabi, S. W. (2020). Mapping the situation of research on coronavirus disease-19 (COVID-19): a preliminary bibliometric analysis during the early stage of the outbreak. BMC Infectious Diseases. https://doi.org/10.1186/s12879-020-05293-Z 